\title{
Teoría de los juegos y su aplicación a los negocios internacionales: acuerdo comercial Unión Europea- Ecuador en el sector agrícola bananero
}

\section{Theory of games and its application to international business: European Union-Ecuador trade agreement in the banana sector}

Econ. Jackson Jiménez Cabrera

Tecnológico Almirante Illingworth, Ecuador

Autor para correspondencia: jacksonjimenez26@yahoo.es

Fecha de recepción: 03 de agosto de 2018 - Fecha de aceptación: 01 de diciembre de 2018

\section{Resumen}

El banano en el Ecuador representa el 10\% de las exportaciones totales; y es el segundo rubro de mayor exportación. La adecuada calidad y el buen sabor que posee el banano ecuatoriano le han permitido posicionar al Ecuador a nivel internacional como uno de los mayores exportadores, ya que su fruta llega en su gran mayoría a los países importadores, en especial del mercado europeo; permitiéndole oportunidades de realizar acuerdos comerciales. Sin embargo, las negociaciones comerciales internacionales del banano ecuatoriano no han sido un tema fácil; sus aristas han estado envueltas de infinidad de intereses, conflictos y disputas legales, lo que en ocasiones le ha generado desventajas comerciales en condiciones discriminatorias. Por medio de la teoría de los juegos se pretende analizar los conflictos, tratando de identificar las causas del mismo, las estrategias de los jugadores, el resultado alcanzado y se tomen decisiones asertivas en los futuros escenarios de las políticas comerciales en el ajedrez de la economía mundial.

Palabras clave: teoría de los juegos; negocios internacionales; acuerdos comerciales

\begin{abstract}
Bananas in Ecuador represent $10 \%$ of total export; and it is the second largest export item. The adequate quality and good taste of Ecuadorian bananas has allowed Ecuador to position itself internationally as one of the largest exporters, since its fruit comes mostly to importing countries, especially the European market, allowing you opportunities to make commercial agreements. However, the international trade negotiations of Ecuadorian bananas have not been an easy subject; its edges have been surrounded by infinity of interests, conflicts and legal disputes, which in some cases has generated commercial disadvantages in discriminatory conditions. Through the theory of game, the aim is to analyze the conflicts, trying to identify the causes of the conflicts, the strategies of the players, the result achieved and making assertive decisions in the future scenarios of commercial policies in the chess of the economy world.
\end{abstract}

Key words: theory of game; international business; trade agreements 


\section{Introducción}

La Constitución de la República del Ecuador del 2008 en su sección séptima, articulo 304, indica que uno de los objetivos de la política comercial es regular, promover y ejecutar las acciones correspondientes para impulsar la inserción estratégica del país en la economía mundial. Por consiguiente, el cambio de la Matriz Económica Productiva Nacional; y el Plan Nacional de Desarrollo para el Buen Vivir 2013- 2017, se estableció como uno de sus objetivos el profundizar una política comercial estratégica y soberana, articulada al desarrollo económico y social del país, bajo estos y otros referentes, es imprescindible promover negociaciones comerciales en condiciones de comercio justo en el marco de acuerdos enfocados en el desarrollo y promocionando a los sectores potenciales; esto sin olvidar de proteger a los sectores más vulnerables.

El campo de acción de la teoría de los juegos es muy amplio y su aplicación se encuentra en las ciencias sociales; y en ámbitos tan variados como las relaciones o negocios internacionales (Aguiar, Barragán y Lara, 2008). Esta teoría desarrolla herramientas que tienen como objetivo analizar comportamientos (económicos, sociales, etc.) en forma de juegos de estrategias, mostrar que los individuos, las empresas e incluso los países son interdependientes, y que están interesados en encontrar un equilibrio que permita que sus interacciones resulten beneficiosas para todas las partes (Fernández, 2010).

Objetivo de la investigación

Analizar, la teoría de los juegos y su aplicación cuantitativa a un modelo hipotético de negocios internacionales del sector bananero.

Materiales y métodos

El presente trabajo investigativo comprende inicialmente un análisis sobre la teoría de los juegos y las principales estrategias utilizadas. Seguido, se realiza una breve explicación sobre las relaciones comerciales entre la Unión Europea y la República del Ecuador, el pasado, presente y futuro de los acuerdo comerciales en el ámbito agrícola, específicamente del sector bananero, que serán expuestos en forma resumida.

Por último, utilizando metodología cuantitativa se planteará un modelo hipotético donde se aplicará teoría de los juegos; en las negociones comerciales internacionales entre la Unión Europea y el Ecuador en el comercio del banano.

Las fuentes de información bibliográficas están basadas en textos relacionados a la teoría de los juegos y los negocios internacionales, además de consultar la dirección electrónica de los principales diarios y leyes ecuatorianas, además de instituciones públicas como el Banco Central del Ecuador para información relacionada a comercio exterior, negociaciones y acuerdos comerciales del banano.

\section{Resultados y discusión}

\section{Teoría de los juegos, decisiones estratégicas y los negocios internacionales}


Un juego es una situación en la que los jugadores (los participantes) toman decisiones estratégicas es decir, decisiones que tienen en cuenta las acciones y respuestas de los demás. Las decisiones estratégicas reportan ganancias o pérdidas a los jugadores: resultados que generan recompensas, beneficios o sanciones (Pérez, Jimeno y Cerda, 2004).

Un objetivo clave de la teoría de los juegos es averiguar la estrategia óptima para cada jugador. Una estrategia es una regla o plan de acción para jugar. La estrategia óptima para un jugador es la que maximiza su ganancia esperada (Avinash y Nalebuff, 1991).

Según Gibbons (1992), algunas estrategias pueden tener éxito si los competidores eligen determinadas opciones; pero fracasan si eligen otras. Sin embargo, otras pueden tener éxito, independientemente de lo que hagan los competidores. están:

(Pindyck \& Rubinfiel, 2001).Entre las principales estrategias en la teoría de los juegos

- La Estrategia Dominante, es aquella que es óptima e independiente de lo que haga el adversario, nos ayuda a averiguar cómo la conducta racional de cada jugador conduce a una solución de equilibrio.

El siguiente ejemplo nos ilustra este concepto. Supongamos que las empresas A y B venden productos rivales y tienen que decidir si emprenden o no una campaña publicitaria. La decisión que tome cada una afectará a la de la otra.

La siguiente matriz de pagos muestra los posibles resultados del juego:

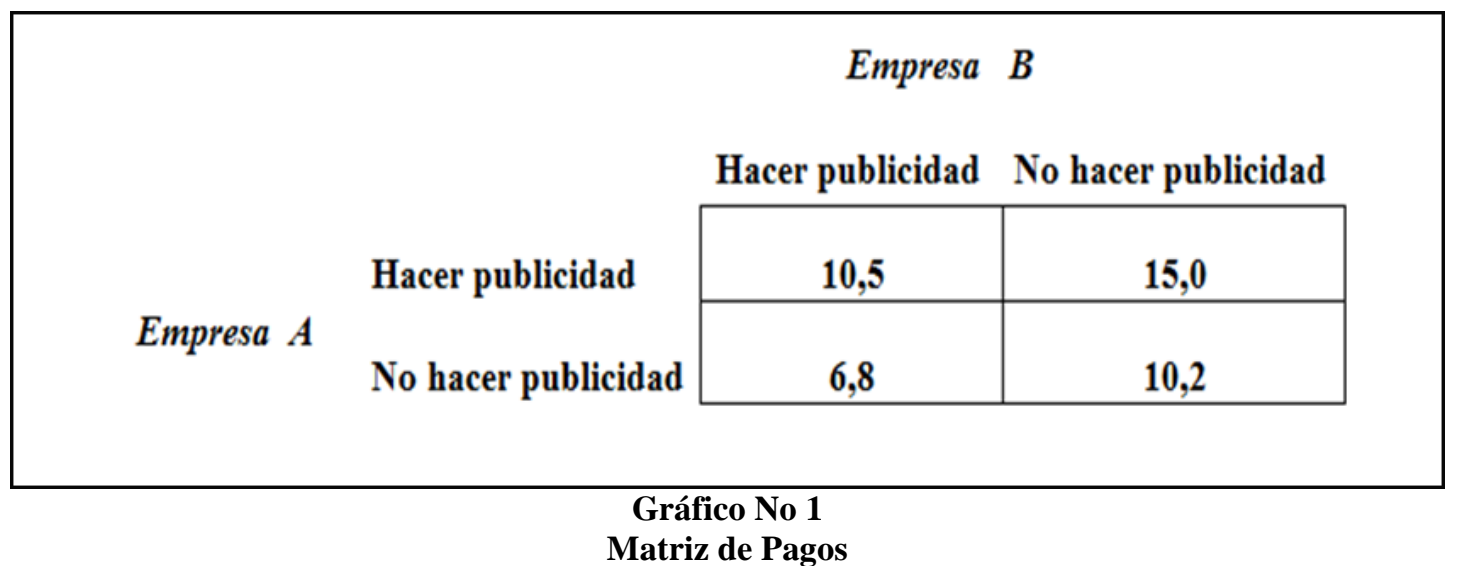

Tomado de: Pindyc \& Rubienfeld. Microeconomía.5ta. edición. p.479

Hacer publicidad es una estrategia dominante para la empresa A. lo mismo ocurre con la $\mathrm{B}$; independientemente de lo que haga la $\mathrm{A}$, lo mejor para la $\mathrm{B}$ es hacer publicidad. Por lo tanto suponiendo que las dos empresas son racionales, sabemos que el resultado de este juego es que ambas empresas harán publicidad. Por lo tanto, suponiendo que las dos empresas son racionales, sabemos que el resultado de este juego es que ambas empresas harán publicidad. Cuando cada jugador tiene una estrategia dominante, llamamos al resultado del juego equilibrio en estrategias dominante. 
- Equilibrio de Nash, es un conjunto tal de estrategias (o actos) que cada jugador hace lo mejor para él, dado lo que hacen sus adversarios. El equilibrio de Nash ocurre cuando el jugador A elige la mejor acción posible, dada la acción del jugador B, y el jugador B elige la mejor acción posible, dada la acción del jugador A.

El ejemplo clásico en la teoría de juegos es el llamado dilema del prisionero, que ilustra el problema al que enfrentan las empresas oligopolistas.

El dilema se ilustra en la siguiente matriz en la cual se consideran las consecuencias de confesar y no confesar sobre dos prisioneros que realizaron el asalto a un banco.

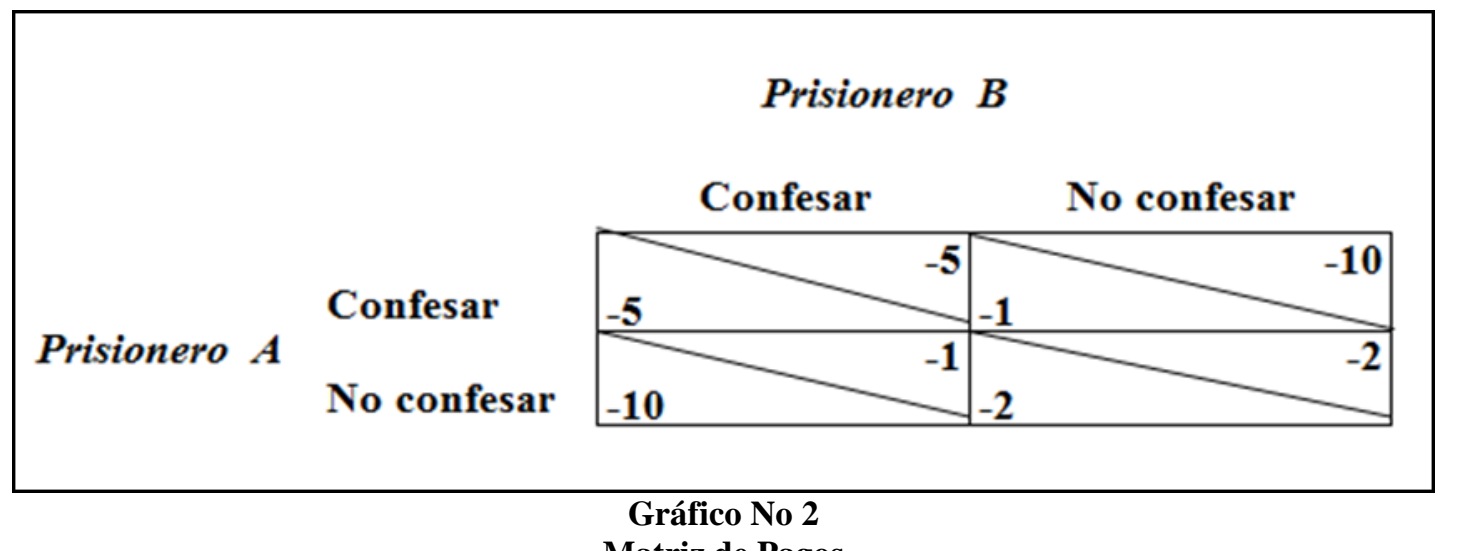

Tomado de: Pindyc \& Rubienfeld. Microeconomía.5ta. edición. p.484

En el dilema de los prisioneros, no importa lo que haga el prisionero A, la mejor estrategia del prisionero B es confesar; y sin importar lo que haga el prisionero B, la mejor estrategia del prisionero A es confesar. Cada prisionero entiende que independientemente de lo que haga el otro, su mejor acción es confesar, cada uno lo hace y recibe una condena de 5 años de cárcel; y así el fiscal de distrito ha resuelto el asalto al banco. Entonces, el equilibrio del dilema de los prisioneros es que cada jugador confiese.

En el resultado probable de un juego, se buscan estrategias "indiscutibles" o "estables". Las estrategias dominantes son estables, pero en muchos juegos uno o más jugadores carecen de una estrategia dominante, por lo que se necesita de un equilibrio general aplicable e intuitivamente atractivo como es el equilibrio de Nash.

\section{Relaciones Comerciales entre la Unión Europea y la República del Ecuador.}




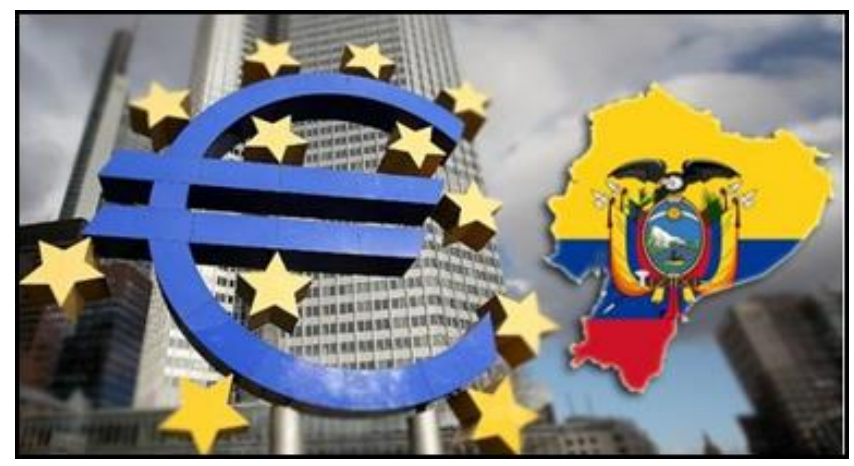

La Unión Europea (UE) se ha consolidado como el grupo económico más importante del mundo; y el principal comprador de bienes y servicios a nivel mundial.

En su conjunto, los países de la UE, representan el mayor mercado, con alrededor de 514 millones de habitantes (Eurostat, 2017), de grandes oportunidades, con consumidores que valoran la calidad, la responsabilidad social y el trabajo de los pequeños productores; ámbitos en los que el Ecuador ha puesto particular empeño en desarrollar y promover en sus negociaciones comerciales.

Las relaciones comerciales de Ecuador con la UE, se han desarrollado bajo el Sistema General de Preferencias Arancelarias, o GSP Plus, por sus siglas en inglés (Generalised System of Preferences), que la Unión Europea le concede al Ecuador de manera unilateral. El Ecuador se encuentra dentro de las categorías de países incluidos en el régimen especial de estímulo del desarrollo sostenible y la gobernanza, y que se encuentran sujetas a las normas de la Organización Mundial de Comercio (OMC). Dentro de la OMC el Ecuador recibe el trato de nación más favorecida (NMF).

\section{Evolución de la Balanza Comercial Ecuador -Unión Europea}

Durante el período de análisis (2010 - 2015), como se observa en el grafico No 3, el Ecuador ha registrado diversos comportamientos de superávit de balanza comercial con la UE. Las exportaciones y las importaciones crecieron en promedio $3.7 \%$ y $6.0 \%$ respectivamente, durante el período referido. 


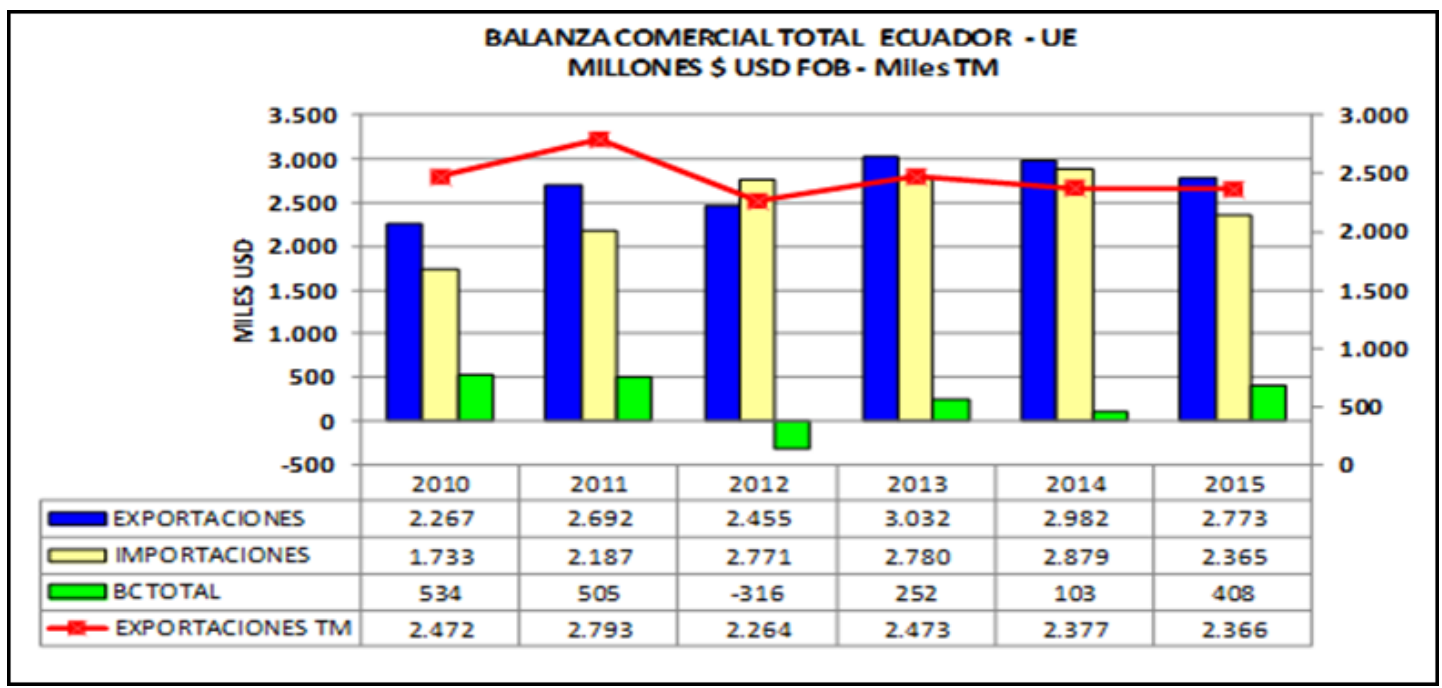

Gráfico No 3

Fuente: COMTRADE y Banco Central del Ecuador, Sección estadísticas de comercio exterior Elaboración: El autor

Las exportaciones ecuatorianas a la UE con relación al total de exportaciones representaron el $13.9 \%$ y $17.1 \%$ para los años 2010 y 2015, respectivamente. La participación de las importaciones ecuatorianas desde la UE con relación al total de las importaciones representó $11.7 \%$ y $16.1 \%$ para los años analizados 2010 y 2015 respectivamente.

(Comtrade y Banco Central del Ecuador, 2017) Los principales productos de exportación de Ecuador a la Unión Europea son: banano, frutas y hortalizas frescas, camarones y langostinos, conservas de atún y demás pescados, cacao y chocolates, extracto y esencia de café, confites, rosas y flores, conservas de frutas, de vegetales y de hortalizas, aceite de palma, jugos de frutas tropicales y de palmito, confecciones, calzado, sombreros, manufacturas plásticas y artesanías.

Entre las principales importaciones desde la Unión Europea al Ecuador se encuentran los bienes de capital para la industria, materias primas y productos intermedios para la industria, materiales de construcción, combustibles y lubricantes, productos conexos, equipos de transporte, bienes de capital para la agricultura y bienes de consumo no duradero.

Negociaciones comerciales en el ámbito agrícola entre la Unión Europea y Ecuador: Caso banano

Las negociaciones comerciales del tratado con la Unión Europea se las puede considerar beneficioso al generar la creación de comercio, es decir los países podrán comprar bienes donde sean menos costosos y vender sus principales productos de exportación. Así mismo producirá ganancias no tradicionales, al permitir el crecimiento de los países por efectos de la inversión nacional como extranjera, transferencia de tecnología por el comercio con países desarrollados y fortalecimiento de la plataforma institucional.

Sin embargo, se presentan riesgos, para los países en desarrollo dentro de los cuales se destacan, el acceso a los mercados, cuyas reglas de origen obstaculizan el intercambio de mercancías en la agricultura, el uso de medidas sanitarias, fitosanitarias arbitrarias, trato diferenciado arancelario, y subsidios a exportaciones agrícolas de parte de los países 
industrializados. Otro de los riesgos que enfrenta el sector agropecuario ecuatoriano, y que en ocasiones se ven agudizados, es por los diferenciales cambiarios internacionales; ya que el Ecuador mantiene el sistema monetario de dolarización, lo que profundiza la pérdida del nivel de competitividad de sus subsectores productivos, y por la falta de medidas de política estatales de largo plazo de apoyo al sector hacen difícil mantener en las mismas condiciones una ventaja competitiva en el mercado internacional; más aún si le agregamos el efecto de competencia desleal que provocan los productos subsidiados por los países desarrollados que presionan a que los precios pagados a los productores agropecuarios se mantengan con una tendencia a la baja.

\section{Pasado, presente y futuro de las negociaciones de acuerdos comerciales de banano entre Ecuador y la Unión Europea}

Los más de 20 años de "guerra del banano" entre la Unión Europea y América Latina fue supuesta como una de las disputas más prolongadas en el tiempo en el marco del sistema multilateral de comercio nacido tras la II Guerra Mundial.

Bruselas y 11 países latinoamericanos (Brasil, Colombia, Costa Rica, Ecuador, Guatemala, Honduras, México, Nicaragua, Panamá, Perú y Venezuela) firmaron en noviembre del 2012 en la Organización Mundial de Comercio el documento que ratificó la paz tras 21 años de conflicto.

La disputa legal se inició en 1993 y estaba dada a causa del diferente trato arancelario que Bruselas concedía al banano que importaba de América Latina y de los países de África, Caribe y Pacifico (ACP), cuando ese grupo de países anuncio el cambio de sistema de licencias de importación, por otro arancelario, que discriminaba a la fruta latinoamericana.

Actualmente, en los mercados internacionales de Europa, Asia y América del Norte el banano ecuatoriano es reconocido por su calidad y sabor, atributos que le han permitido ocupar el $30 \%$ de la oferta mundial y posicionar al Ecuador como el mayor exportador en el mundo (Catalogo V Cumbre Mundial del Banano, 2004).

Por consiguiente, la firma del Acuerdo Comercial Multipartes; lograda en diciembre del 2016 con la Unión Europea le ha permitido al Ecuador mantener su liderazgo como país exportador de banano en el mercado europeo, según información de las cumbres mundial de banano. Sin embargo, desde el inicio del Acuerdo Comercial (enero del 2017) durante tres años consecutivos la Unión Europea podrá aplicar varios mecanismos para proteger a sus productores agrícolas de las importaciones provenientes desde Ecuador (Agencia AFP El Telégrafo, 2017, noviembre 30 ).

El alcance de las medidas es que si durante un año o menos Ecuador supera cierto umbral de exportaciones de cajas de banano, la UE podrá cobrar un arancel como si no hubiese un acuerdo comercial. Considerando, que esto solo se podrá aplicar en los tres primeros años de vigencia del acuerdo. El umbral de exportaciones para el año 2017 se fijó en 1'801.788 toneladas, cifras que van creciendo hasta alcanzar las 1'957.500 toneladas en el año 2019, y la alarma temprana para los europeos podría activarse cuando las exportaciones de Ecuador alcancen el 80\% de esas cifras. Este mecanismo, es aplicado hasta el año 2019 y a partir del 
2020, ya no se aplicará; y el banano ecuatoriano será uno de los principales beneficiaros del acuerdo comercial.

Aplicación de la teoría de los juegos en las negociaciones de acuerdos comerciales internacionales entre la Unión Europea y Ecuador en el ámbito del sector bananero

Analizados los conceptos de la teoría de los juegos y según Jhon Von Neuman que propone una concepción del mundo como si fuera un juego matemático; un mundo regido por una lógica universal en el que la persona posee y actúa como un ser perfectamente lógico que trata con la realidad circundante intercambiando información matemática (Sierralta, 2009). Se pasa a la ejemplificación hipotética de un modelo aplicando metodología cuantitativa, para ello se señalan las principales reglas del juego, desarrollo de las negociaciones, indicando las jugadas estratégicas, número de jugadores, solicitudes de cada jugador y beneficios que permita alcanzar un acuerdo en equilibrio para ambas naciones.

El siguiente es un juego no cooperativo ya que los jugadores ( Unión Europea República del Ecuador) no pueden establecer acuerdos vinculantes, es decir no estipulan convenciones con otros jugadores y más bien diseñan su propia estrategia la que maximiza su beneficio dado las estrategias del otro jugador. Existe información perfecta por parte de cada jugador lo que le permitirá diseñar sus propias estrategias y sus beneficios a partir de las estrategias del otro jugador.

La búsqueda de maximización de la estrategia se adentra en la indagación del equilibrio para no perder frente a las otras estrategias de los otros sujetos, llegando así al equilibrio de Nash.

Se tiene, como referencia, un juego de dos jugadores con las siguientes preferencias:

- Jugador 1 (Unión Europea)

- Jugador 2 ( República del Ecuador)

- Estrategias del juego

- Cooperar conjuntamente a un acuerdo de negociación de apertura total al comercio del banano. ( $\mathrm{CO}=$ coopera con el acuerdo de negociación)

- No coopera conjuntamente a un acuerdo de negociación de apertura total al comercio del banano. ( $\mathrm{NC}=$ no coopera con el acuerdo de negociación)

\section{Estructura de las Solicitudes}

\section{Solicitud de la Unión Europea}

La Unión Europea es el mayor importador mundial de productos alimenticios, por un amplio margen. A través de su política de desarrollo, la UE ayuda a los países en desarrollo a vender sus productos agrícolas en su territorio. Y lo hace concediéndoles un acceso preferencial a su mercado.

Por otro lado, la Política Agraria Común (PAC) de la UE, creada en 1962, representa una asociación entre la agricultura y la sociedad, entre Europa y sus agricultores. La política agrícola 
es uno de los ámbitos en los que los países de la UE aceptaron centralizar plenamente sus competencias, además de la financiación pública necesaria. Por lo tanto, las decisiones y las ayudas no están en manos de cada país, sino que son responsabilidad de la UE en su conjunto. De esta manera, un acuerdo que perjudique a sus agricultores es total y esta presión se traslada directamente a la posición negociadora de la Unión Europea.

\section{Solicitud de la República del Ecuador}

Con una exportación sobre el 30\% de la oferta mundial, el Ecuador sigue siendo el mayor exportador de banano en el mundo; llegando a todos los países importadores de la fruta. El banano representa el $10 \%$ de las exportaciones totales y el segundo rubro mayor de las exportaciones ecuatorianas, al ser una fruta apetecida por consumidores de los mercados más exigentes y formar parte de la dieta diaria de millones de personas. La actividad bananera genera fuentes de trabajo e ingresos a 2 millones de personas involucradas en las diferentes etapas de su cadena de valor.

El Ecuador ha considerado discriminatorio los aranceles aplicados por la Unión Europea que en negociaciones anteriores le han aplicado a su fruta; esto porque a diferencia de los estados ACP (África, Caribe y Pacifico) pueden vender una cuota anual de bananas libres de impuestos, lo que le ha generado pérdidas de mercado a sus productores. Por lo que solicita la posible eliminación total de la cuota arancelaria para la liberalización comercial mundial de la fruta.

\section{Análisis del juego}

Considerando hipotéticamente que las negociaciones entre la Unión Europea y Ecuador se realizarán en un corto período; se resumen los pagos o recompensas en la siguiente matriz.

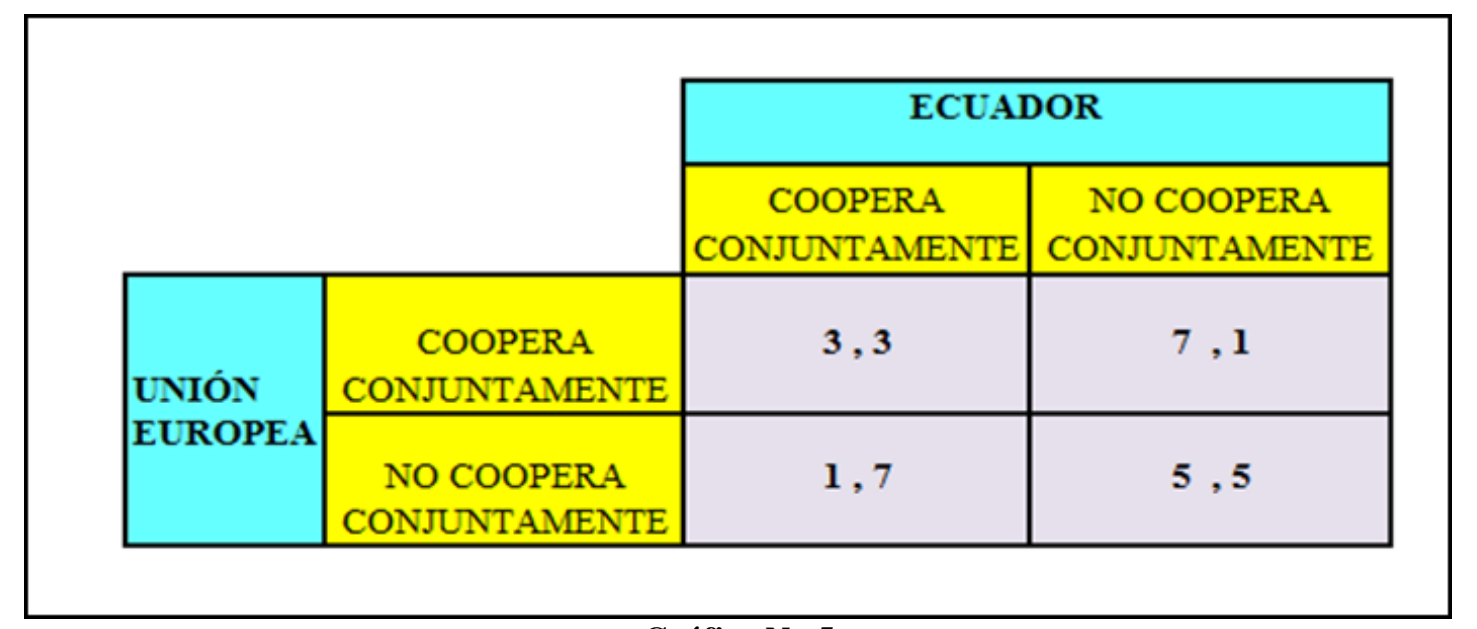

Juego de Negociación del Banano

Fuente: Elaborado por el autor

Analizando la estrategia del juego desde el punto de vista de la República del Ecuador. Ecuador se da cuenta que su resultado depende de las decisiones que tome la Unión Europea. Si 
la UE coopera conjuntamente en un acuerdo de negociación a Ecuador también le conviene cooperar realizándose el acuerdo en un plazo de 3 años, en vez de que Ecuador en un año pueda realizar un acuerdo de negociación pero sin lograr una cooperación conjunta.

Por consiguiente, si la UE en un año no coopera a un acuerdo de negociación conjunta, 7 años le costará alcanzar un acuerdo de cooperación conjunta a Ecuador, en lugar de 5 años y sin lograr acuerdos de cooperación. El dilema, desde el punto de vista de La Unión Europea, es idéntico al de Ecuador. Consecuentemente, Ecuador analiza su jugada y concluye que independientemente de lo que haga la Unión Europea, lo mejor que le conviene es un acuerdo de negociación en cooperación conjunta.

A los dos jugadores les interesa llegar a un arbitraje de negociación siempre y cuando no se consideren políticas comerciales restrictivas a sus productores de lo contrario no cooperan conjuntamente a una negociación. Cada nación entiende que independientemente de lo que haga el otro, su mejor acción es negociar en cooperación conjunta, haciéndolo en 3 años con la aplicación de una política comercial sin medidas discriminatorias o restrictivas y con un estilo negociador analítico cooperativo; siendo este es el equilibrio (Nash) del juego.

Resumiendo las estrategias de la matriz los pagos con la nomenclatura correspondiente

$$
\mathrm{CO}=\text { coopera conjuntamente } / \mathrm{NC}=\text { no coopera conjuntamente }
$$

En función de los resultados el equipo negociador puede llegar a un acuerdo de negociación conjunta aproximándose a la función $(\mathrm{CO}=3, \mathrm{CO}=3)$; caso contrario no se llega a una negociación en cooperación conjunta y será la función $(\mathrm{NC}=5, \mathrm{NC}=5)$.

Por último, el debate comercial indica la discusión en cuanto se abre o no al libre comercio la Unión Europea ante la solicitud del Ecuador. De acuerdo a los resultados del juego, les conviene a las naciones una negociación en cooperación conjunta caso contrario se llega a una negociación sin una verdadera cooperación, y no hay lugar para un nuevo arbitraje sobre la materia lo que deja al bloque europeo con las opciones de seguir con un menor arancel y eliminarlo en su totalidad a partir del 2020 según el Acuerdo Comercial Multipartes firmado en el 2016; o seguir presionando y arriesgarse a fuertes sanciones de parte de la Organización Mundial de Comercio.

\section{Conclusiones y recomendaciones}

Se concluye que, la teoría de los juegos, facilita el elemento objetivo para que las partes interactúen y lleguen a un acuerdo, resolver un conflicto, o desarrollar un proyecto conjunto y calcular así el riesgo. Desconociendo si el resultado final sea cual fuere; si el desastre o la ruina para el que pierde o la ganancia o beneficio del que gana. En el caso del modelo propuesto se ha evidenciado al final del juego un arbitraje de estrategias en el, que han tenido en cuenta la información básica de las partes, el escenario, el momento del juego y donde ambos jugadores obtendrán beneficios. 
La UE debe entender que es posible que unos ganen más que otros, pero que no siempre en detrimento de los países latinoamericanos que se encuentran en vías de desarrollo, en donde aún persisten problemas sociales y de extrema pobreza.

Debido a que la teoría de los juegos ha tenido un rol protagónico en grandes cambios que han experimentado las ciencias sociales; específicamente la economía, se recomienda aplicarla para el resto de ciencias.

\section{Bibliografía}

Aguiar, F., Barragán, J. y Lara, N. (2008). Economía, sociedad y teoría de juegos. Barcelona, España: Editorial McGraw -Hill.

Avinash, D. y Nalebuff, B. (1991). Pensar Estratégicamente. España: Editor Antoni Bosch Recuperado de:

https://books.google.com.ec/books?id=z4ftialL7JQC\&printsec=frontcover\&hl=es\&sourc $\mathrm{e}=\mathrm{gbs} \_g e \_s u m m a r y \_r \& c a d=0 \# \mathrm{v}=$ onepage $\& \mathrm{q} \& \mathrm{f}=\mathrm{false}$.

Agencia AFP. (2017, Noviembre 30). UE considera "positivo "su acuerdo comercial con Ecuador. El Telégrafo. Recuperado de: http://www.eltelegrafo.com.ec/noticias/economia/1/ue-considera-positivo-su-acuerdocomercial-con-ecuador

Banco Central del Ecuador. (2017). Sección estadísticas de comercio exterior.

Recuperado de www.bce.fin.ec

Base de datos Comtrade de la ONU. (2017). Sección estadísticas de comercio internacional. Recuperado de https://comtrade.un.org/

Base de datos Eurostat. (2017). Sección estadísticas de comercio internacional.

Recuperado de: https://translate.google.com.ec/translate?hl=es\&sl=en\&u=http://ec.europa.eu/eurostat\&pr ev=search.

Constitución de la República del Ecuador. (2008). Quito, Ecuador: Ediciones legales.

Expo plaza. (2014). Catálogo oficial de la V Cumbre Mundial del Banano 2014. 1 (1). Edición especial.

Fernández, J. (2010). Teoría de Juegos: Su aplicación en economía (2da ed.). México: Editorial Centro de Estudios Económicos de México.

Gibbons, R. (1992). Un primer curso de teoría de juegos. Barcelona, España: Editor Antoni Bosch.

Pérez, J., Jimeno, J. y Cerdá, E. (2004). Teoría de Juegos. Madrid, España: Pearson 


\section{Educación.}

Pindyc, R., y Rubinfeld, D. (2001). Microeconomía (5ta ed.). Madrid, España: Pearson Educación.

Plan Nacional del Buen Vivir 2013-2017. (2017). Ecuador: Recuperado de: https://www.unicef.org/ecuador/Plan_Nacional_Buen_Vivir_2013-2017.pdf

Redacción economía. (2017, Mayo 11). Las exportaciones a la UE aumentaron \$100 Millones. El Telégrafo. Recuperado de http://www.eltelegrafo.com.ec/noticias/economia/8/las-exportaciones-a-la-ueaumentaron-usd-100-millones

Sierralta, A. (2009). Negociaciones y Teoría de los Juegos. Perú: Fondo editorial Pontificia Universidad Católica del Perú. 